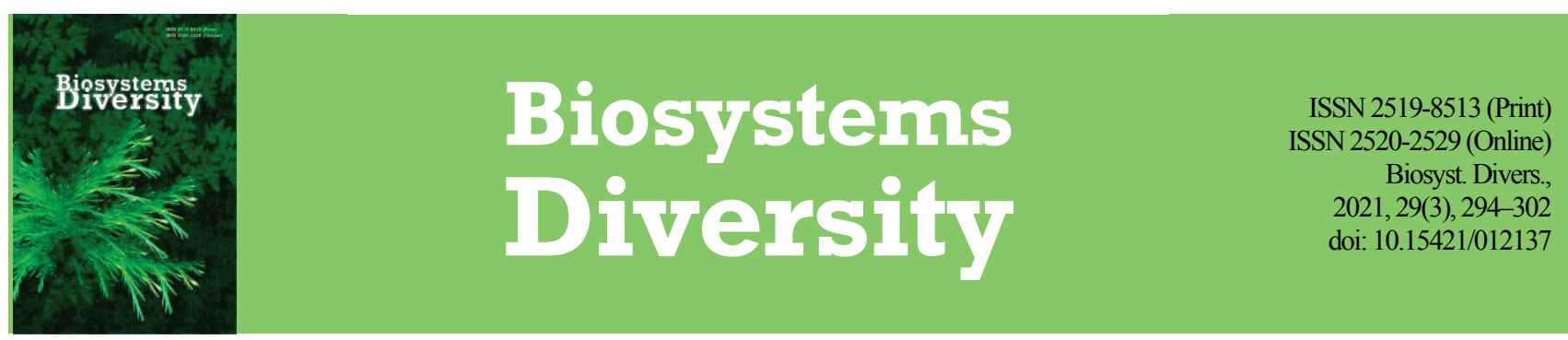

\title{
A systematic review of flukes (Trematoda) of domestic goose (Anser anser dom.)
}

\author{
F. H. Rzayev \\ Azerbaijan Medical University, Baku, Azerbaijan \\ Institute of Zoology of ANAS, Baku, Azerbaijan
}

Article info

Received 01.07.2021

Received in revised form 02.08 .2021

Accepted 04.08.2021

Azerbaijan Medical

University, Nasimi reg.,

Samad Vurgun st., 163 ,

Baku, AZ1078, Azerbaijan.

Tel.: +99-412-597-38-98.

E-mail:fuad.zi@mail.nu

Institute of Zoology, ANAS,

A.Abbaszadeh st.,

passage 1128, block 504

Baku, AZ1004, Azerbaijan.

Tel.: +99-412-539-73-71.

E-mail:

fuad.rzayev01f@gmail.com

\section{Rzayev, F. H. (2021). A systematic review of flukes (Trematoda) of domestic goose (Anser anser dom.). Biosystems Diversity, 29(3), 294-302. doi:10.15421/012137}

Trematodes are one of the most numerous and widespread groups of parasitic invertebrates among helminths, characterized by a complex life cycle. As the final host, digenetic flukes parasitize vertebrates, including domestic waterfowl. Infection of hosts with parasitic digenetic worms causes huge economic damage to poultry. But trematode fauna of domestic geese are presented mainly only for individual regions. Despite the fact that there is already a systematic review of tapeworms and acanthocephala of domestic birds - geese and ducks, there are, unfortunately, no review articles on the taxonomy of digenetic flukes for geese (Anser anser $\mathrm{dom}$.). Taking into account the relevance of the topic, based on our own and extensive literature data, we set the task to determine the general species composition of digenetic flukes (Trematoda), parasitizing in domestic geese which have been recorded in the world until 2020. Each Trematoda species is provided with the following data: scientific name, authority and year, first, second intermediate, auxiliary and final hosts, site in the host body, collecting localities and geographic distribution, and literature sources. Currently it is established that there are 149 species of helminths parasitising domestic geese. The taxonomic composition of the class Trematoda registered in domestic geese consists of 2 classes, 14 families, 30 genera and 65 species. Of all the species of trematodes parasitizing in domestic geese, 44 species have been recorded in Europe, 26 species in Asia, 6 species in North America, 1 species in South America, 1 species in Africa and 2 species in Oceania. Three species are registered in domestic geese (Echinoparyphium recurvatum, Echinostoma revolutum, Prosthogonimus ovatus) are cosmopolitan parasites. The biggest species diversity is characterized by the families Echinostomatidae (17 species) and Notocotylidae (12 species). There are 11 species of digenetic flukes recorded in domestic geese Cyclocoelum mutabile, Echinostoma grande, E. paraulum, E. revolutum, E. robustum, Hypoderaeum conoideum, Paryphostomum novum, P. pentalobum, Catatropis verrucosa, Notocotylus attenuatus, $N$. parviovatus) on the territory of Azerbaijan. Four of them (Echinostoma revolutum, Hypoderaeum conoideum, Paryphostomum novum, Notocotylus attenuatus) were also noted in the course of our helminthological studies. The trematode Cyclocoelum mutabile was recorded in domestic ducks only in Azerbaijan. Most species of trematodes were found in the north-eastern part (total 9 species) and southern part (total 5 species) of Azerbaijan. This is due to the widespread distribution of molluscs - intermediate hosts of digenetic flukes, in these regions. Eighteen species of digenetic flukes parasitize both birds and mammals (Rodentia, Carnivora, Lagomorpha, Artiodactyla). And eleven species of trematodes are of medical importance, registered in humans.

Keywords: fauna; systematic classification; helminthes, water bird, Azerbaijan.

\section{Introduction}

Trematodes, a class of digenetic flukes, being biohelminths, have the largest number of species among helminths in wetland birds, including domestic waterfowl. Infection of hosts with parasitic digenetic worms causes huge economic damage to poultry. The study of the species composition of bird helminths, including trematodes of domestic geese (Anser anser dom.) has been carried out in some countries (Lapage, 1961; McDonald, 1969; Smogorzhevskaya, 1976; Sitko et al., 2006; Shakarbayev et al., 2012; Kirillov \& Kirillova, 2013; Berenice et al., 2014), including in Azerbaijan (Shirinov, 1961; Vahidova, 1978). In recent years (2005-2020), on the territory of the republic, the helminth fauna of domestic waterfowl and some ecological aspects have been studied by us (Rzayev, 2008, 2013; Rzayev \& Ibrahimova, 2015; Seyidbeyli \& Rzayev, 2018; Rzayev et al., 2020; Seyidbeyli et al., 2020). However, it should be noted that data on the trematode fauna of domestic geese are presented mainly for individual regions. Despite the fact that there is already a systematic review of tapeworms and acanthocephala of domestic birds geese and ducks (Rzayev, 2021; Rzayev et al., 2021; Rzayev \& Gasimov, 2021), there are unfortunately no review articles on the taxonomy of digenetic flukes for the above birds. Taking into account the relevance of the topic, based on our own and literature data, we set the task to determine the general species composition of digenetic flukes (Trematoda), parasitizing in domestic geese which have been recorded in the World until 2020.

\section{Material and methods}

In addition to the extensive literature data, we carried out a parasitological study to determine the helminth fauna of domestic waterfowl, including domestic geese, in the territory of Azerbaijan. In total, 470 domestic geese from private poultry farms in various regions of the Republic were researched by the method of complete parasitological examination (Skrjabin, 1928; Dubinina, 1971). The collected flukes were stained with carmine and dehydrated, and then permanent preparations were prepared with Canadian balsam. The preparations were studied under a Primo Star light microscope (Carl Zeiss, Germany). We used an identification guide for species identification (Ryzhikov, 1967). In total, 21 species of helminths were identified in geese. Of these, 4 species are digenetic flukes.

The Trematoda list is arranged following the nomenclature and classification from three volumes of books "Keys to Trematoda" by Gibson et al. (2002), Jones et al. (2005), Bray et al. (2008), with phylogenetic updates by Olson et al. (2003). We also took into account the classification adopted by the database of World Register of Marine Species (WoRMS, www.marinespecies.org). Each Trematoda species is provided with the following data: scientific name, authority and year, first, second intermediate, auxiliary and final hosts, site in host body, collecting localities and geographic distribution, prevalence (PI) and intensity (II) of infection and literature sources. 


\section{Results}

Based on our own research and analysis of extensive literature data, 149 species of helminths have been established today among domestic geese. The taxonomic composition of the class of trematodes (Trematoda) registered in domestic geese consists of two classes (Diplostomida -9 and Plagiorchiida - 56), 14 families (Strigeidae - 4, Cyathocotylidae - 2, Schistosomatidae - 3, Cyclocoelidae - 2, Echinochasmidae - 1, Echinostomatidae - 17, Philophthalmidae - 4, Psilostomidae - 7, Typhlocoelidae1, Heterophyidae - 3, Opisthorchiidae - 4, Notocotylidae - 12, Zygocotylidae - 1, Prosthogonimidae - 4), 30 genera and 65 species. These species are presented below according to the above taxonomy:

Phylum: Platyhelminthes Gegenbaur, 1859

Class: Trematoda Rudolphi, 1808

Subclass: Digenea Carus, 1863

Order: Diplostomida Olson, Cribb, Tkach, Bray, Littlewood, 2003

Family: Strigeidae Railliet, 1919

Genus: Apatemon Szidat, 1928

1. Apatemon gracilis (Rudolphi, 1819) Szidat, 1928

First intermediate hosts: Mollusca - Gastropoda.

Second intermediate hosts: Annelida - Hirudinea; Pisces - Cyprinidae, Salmonidae, Gasterosteidae, Nemachellidae.

Final hosts: Aves - Anseriformes, Charadriiformes, Columbiformes. Site in host: Small intestine.

Locality: Georgia and Kazakhstan (Petrochenko \& Kotelnikov, 1976), China - Zhenjiang, Jiangsu Province (Liu et al., 2018), Russia Amur, Krasnoyarsk regions, mouth of the Volga River (Ryzhikov, 1967; Petrochenko \& Kotelnikov, 1976; Kirillov \& Kirillova, 2013), Slovakia and Czech Republic (PL 1.5\%) (Busta, 1980).

Distribution: Europe, Asia, Africa, North and South America.

Genus: Cotylurus Szidat, 1928

2. Cotylurus cornutus Rudolphi, 1808

First intermediate hosts: Mollusca-Gastropoda.

Second intermediate hosts: Annelida - Hirudinea; Mollusca - Gastropoda.

Final hosts: Aves - Anseriformes, Falconiformes, Galliformes, Charadriiformes, Columbiformes, Passeriformes, Mammalia - human.

Site in host: Small intestine.

Locality: Ukraine - Danube Delta, upper and lower Dniester (Smogorzhevskaya, 1976; Iskova et al., 1995), Russia - Rostov and Moscow regions (Ryzhikov, 1967; Petrochenko \& Kotelnikov, 1976), Slovakia and Czech Republic (PL 0.1\%) (Busta, 1980).

Distribution: Europe, Asia, Africa, North and South America.

3. Cotylurus flabelliformis Faust, 1917

First and second intermediate hosts: Mollusca-Gastropoda.

Final hosts: Aves - Anseriformes, Galliformes.

Site in host: intestine, caecum.

Locality: USA (McDonald, 1969).

Distribution: Europe, Asia, North America.

Genus: Ichthyocotylurus Odening, 1969

4. Ichthyocotylurus pileatus (Rudolphi, 1802) Odening, 1969

First intermediate hosts: Mollusca-Gastropoda.

Second intermediate hosts: Pisces - Percidae.

Final hosts: Aves - Anseriformes.

Site in host: Intestine.

Locality: Ukraine (Smogorzhevskaya, 1976).

Distribution: Europe, Asia, North America.

Family: Cyathocotylidae Muhling, 1898

Genus: Cyclocoelum Brandes, 1892

5. Cyclocoelum mutabile (Zeder, 1800) Brandes, 1892

Intermediate hosts: Mollusca - Gastropoda.

Final hosts: Aves - Anseriformes, Galliformes, Gruiformes, Charadriiformes.
Site in host: air sacs, nasal cavity.

Locality: Azerbaijan - Lenkaran region (Vahidova, 1978).

Distribution: Europe, Asia, North America.

6. Cyclocoelum robustum Stossich, 1903

First and second intermediate hosts: unknown.

Final hosts: Aves - Anseriformes.

Site in host: nasal cavity, air sacs.

Locality: Far East (Ryzhikov, 1967; Petrochenko \& Kotelnikov, 1976).

Distribution: Europe, Asia, Australia.

Family: Schistosomatidae Stiles \& Hassall, 1898

Genus: Bilharziella Looss, 1899

7. Bilharziella polonica Kowalewski, 1895

Intermediate hosts: Mollusca-Gastropoda.

Final hosts: Aves - Anseriformes, Podicipediformes, Ciconiiformes, Gruiformes, Charadriiformes.

Site in host: abdominal veins, especially portal veins; intestine, lungs, kidneys.

Locality: Slovakia and Czech Republic (Barus et al., 1977; Sitko et al., 2006), Poland (Bezubik, 1956), Germany (Lapage, 1961), Uzbekis$\tan$ (Shakarbayev et al., 2012), Ukraine (PL 0.1\%, II 1-6) (Smogorzhevskaya, 1976; Iskova et al., 1995), Russia (Ryzhikov, 1967).

Distribution: Europe, Asia, Africa, North America.

Genus: Trichobilharzia Skrjabin \& Zakharow, 1920

8. Trichobilharzia adamsi Edwards \& Jansch, 1955

Intermediate hosts: Mollusca-Gastropoda.

Final hosts: Aves - Anseriformes.

Site in host: liver.

Locality: Canada (McDonald, 1969; Brant \& Loker, 2009).

Distribution: North America.

9. Trichobilharzia oregonensis Macfarlane et Macy, 1946

Intermediate hosts: Mollusca-Gastropoda.

Final hosts: Aves - Anseriformes.

Site in host: portal veins, veins of intestinal and caecum walls.

Locality: USA (Macy et al., 1955; McDonald, 1969).

Distribution: North America.

Order: Plagiorchiida La Rue, 1957

Family: Cyclocoelidae Stossich, 1903

Genus: Hyptiasmus Kossack, 1911

10. Hyptiasmus arcuatus (Brandes, 1892) Kossack, 1911

Intermediate hosts: Mollusca-Gastropoda.

Final hosts: Aves - Anseriformes, Gruiformes, Charadriiformes.

Site in host: nasal cavity, trachea.

Locality: Czech Republic and Slovakia - Presov (Barus et al., 1977;

Sitko et al., 2006), Germany (Lapage, 1961).

Distribution: North America, Europe, Asia.

11. Hyptiasmus laevigatus Kossack, 1911

Intermediate hosts: Mollusca-Gastropoda.

Final hosts: Aves - Anseriformes.

Site in host: nasal cavity, trachea.

Locality: Ukraine and Kazakhstan (Ryzhikov, 1967).

Distribution: North America, Europe, Asia.

Family: Echinochasmidae Odhner, 1910

Genus: Echinochasmus Dietz, 1909

12. Echinochasmus beleocephalus (Linstow, 1873) Dietz, 1909

First intermediate hosts: Mollusca-Gastropoda.

Second intermediate hosts: Amphibia - Pelobatidae, Ranidae; Pisces - Cyprinidae.

Final hosts: Aves - Anseriformes, Ciconiiformes, Galliformes.

Site in host: small intestine, caecum.

Locality: Russia (Ryzhikov, 1967), Ukraine - Danube Delta, Upper

and Lower Dniester (Iskova et al., 1995).

Distribution: Europe, Asia, Africa. 
Family: Echinostomatidae Looss, 1899

Genus: Echinoparyphium Dietz, 1909

\section{Echinoparyphium cinctum Rudolphi, 1802}

First and second intermediate hosts: Mollusca-Gastropoda.

Final hosts: Aves - Anseriformes, Galliformes, Gruiformes, Charadriiformes.

Site in host: intestine.

Locality: Uzbekistan (Shakarbayev et al., 2012), Ukraine (Smogorzhevskaya, 1976), Russia - Omsk and Chelyabinsk regions and Belarus (Ryzhikov, 1967; Petrochenko \& Kotelnikov, 1976).

Distribution: Europe, Asia.

14. Echinoparyphium recurvatum Linstow, 1873

First intermediate hosts: Mollusca-Gastropoda.

Second intermediate hosts: Amphibia - Pelobatidae, Ranidae; Mollusca - Gastropoda.

Final hosts: Aves - Anseriformes, Ciconiiformes, Galliformes, Gruiformes, Charadriiformes, Columbiformes, Strigiformes; Mammalia Rodentia, Carnivora and humans (Lu, 1982).

Site in host: small intestine.

Locality: Ukraine (Iskova et al., 1995; Yuskiv \& Melnychuk, 2020), Russia - Bashkortostan, along the Volga River, Dagestan (Kirillov \& Kirillova, 2013; Marzhokhova \& Zhigunova, 2008), New Zealand (Rind, 1974; Weekes, 1982; McKenna, 2010), Turkey - Marmara (Merdivenci, 1967; Gicik \& Arslan, 2003), Uzbekistan (Shakarbayev et al., 2012), Czech Republic and Slovakia (Sitko et al., 2006).

Distribution: cosmopolitan.

\section{Genus: Echinostoma Rudolphi, 1809}

15. Echinostoma dietzi Skrjabin, 1923

First and second intermediate hosts: Mollusca-Gastropoda.

Final hosts: Aves - Anseriformes, Galliformes, Gruiformes.

Site in host: small intestine.

Locality: Ukraine (Petrochenko \& Kotelnikov, 1976), Uzbekistan (Shakarbayev et al., 2012), Russia - Bashkortostan, along the Volga River, Moscow region (Kirillov \& Kirillova, 2013; Ryzhikov, 1967; Petrochenko \& Kotelnikov, 1976).

Distribution: Europe, Asia.

16. Echinostoma grande Bachkirova, 1946

First and second intermediate hosts: unknown.

Final hosts: Aves - Anseriformes, Gruiformes.

Site in host: small intestine, caecum.

Locality: Russia (McDonald, 1969), Azerbaijan - Shabran region (PL 0.45\%, II 1-6) (Shirinov, 1961).

Distribution: Europe, Asia.

17. Echinostoma lindoense Sandground \& Bonne, 1940

First and second intermediate hosts: Mollusca-Gastropoda.

Final hosts: Aves - Anseriformes, Galliformes; Mammalia - Rodentia and humans (Carney et al., 1980).

Site in host: small intestine.

Locality: Malaysia (McDonald, 1969).

Distribution: Asia.

18. Echinostoma miyagawai Ishii, 1932

First intermediate hosts: Mollusca-Gastropoda.

Second intermediate hosts: Mollusca - Gastropoda; Amphibia - Ranidae.

Final hosts: Aves - Anseriformes, Galliformes, Charadriiformes, Columbiformes; Mammalia - Rodentia and humans (Mao, 1991).

Site in host: small and large intestine. 1967).

Locality: Russia - Bashkortostan, along the Volga River (Ryzhikov,

Distribution: Europe, Asia.

19. Echinostoma paraulum Dietz, 1909

First and second intermediate hosts: Mollusca-Gastropoda.

Final hosts: Aves - Anseriformes, Podicipediformes, Galliformes, Charadriiformes, Columbiformes; Mammalia - humans (Yu \& Mott, 1994).

Site in host: intestine.
Locality: Russia - along the Volga River, North Caucasus, Ural, Amur, Kaluga, Bryansk, Kursk regions (Kirillov \& Kirillova, 2013; Marzhokhova \& Zhigunova, 2008), Kazakhistan and Georgia (Petrochenko \& Kotelnikov, 1976), Azerbaijan - Shabran region (PL 0.6\%, II 1-2) (Shirinov, 1961; Vahidova, 1978).

Distribution: Europe, Asia.

20. Echinostoma revolutum Fröhlich, 1802

First intermediate hosts: Mollusca - Gastropoda.

Second intermediate hosts: Mollusca - Gastropoda, Bivalvia; Amphibia-Bufonidae, Ranidae.

Auxiliary hosts: Pisces - Cyprinidae, Ictalirudae.

Final hosts: Aves - Anseriformes, Podicipediformes, Ciconiiformes, Falconiformes, Galliformes, Gruiformes, Charadriiformes, Columbiformes, Strigiformes, Passeriformes; Mammalia - Rodentia, Carnivora, Lagomorpha, Artiodactyla and humans (Carney, 1991; Sohn et al., 2011).

Site in host: intestine.

Locality: Ukraine (PL. 0.3\%, II 1-13) (Smogorzhevskaya, 1976; Iskova et al., 1995; Yuskiv \& Melnychuk, 2020), Russia - Bashkortostan, Dagestan, Kaluga, Kursk, Ryazan, Tula regions, along the Volga River (Ryzhikov, 1967; Kirillov \& Kirillova, 2013), New Zealand - Rakaia, Coleridge, Ashburton, Banks Peninsula, Waimakariri, Kaikoura, Kaitangata (Rind, 1974; Weekes, 1982; McKenna, 2010), UK (Griffiths et al., 1976), Turkey - Gars and Marmara (Merdivenci, 1967; Gicik \& Arslan, 2003), Czech Republic and Slovakia (Barus et al., 1977; Sitko et al., 2006), Uzbekistan (Shakarbayev et al., 2012), Azerbaijan - Mughan, Lenkaran, Astara, Jalilabad, Bilasuvar, Shabran, Guba, Khachmaz, Shamkir Absheron regions (PL 5.7\%, II 1-21) (Shirinov, 1961; Vahidova, 1978; Aghayeva, 2018), own data-Masally region (PL 0.12\%, II 1).

Distribution: cosmopolitan.

21. Echinostoma robustum Yamaguti, 1935

First intermediate hosts: Mollusca - Gastropoda.

Second intermediate hosts: Mollusca - Gastropoda, Bivalvia; Amphibia-Ranidae.

Auxiliary hosts: Pisces - Cyprinidae.

Final hosts: Aves - Anseriformes, Galliformes, Columbiformes

Site in host: small intestine.

Locality: Ukraine - Black Sea coast (PL 0.03\%, II 13-16) (Smogorzhevskaya, 1976; Iskova et al., 1995), Russia - Bashkortostan, Amur region, along the Volga River (Kirillov \& Kirillova, 2013), Azerbaijan Shabran region (PL 0.9\%, II 1-4) (Shirinov, 1961).

Distribution: Europe, Asia.

22. Echinostoma sudanense Odhner, 1911

First and second intermediate hosts: unknown.

Final hosts: Aves - Anseriformes, Ciconiiformes.

Site in host: small intestine, large intestine.

Locality: Ukraine (Smogorzhevskaya, 1976).

Distribution: Europe, Africa.

Genus: Hypoderaeum Dietz, 1909

\section{Hypoderaeum conoideum Bloch, 1782}

First intermediate hosts: Mollusca-Gastropoda.

Second intermediate hosts: Mollusca-Gastropoda, Bivalvia.

Final hosts: Aves - Anseriformes, Galliformes, Gruiformes, Columbiformes; Mammalia - human (Bhaibulaya et al., 1964; Yokogawa et al., 1965).

Site in host: small intestine.

Locality: Czech Republic and Slovakia (PL 0.1\%) (Busta, 1980; Sitko et al., 2006), Russia - Bashkortostan, along the Volga River (Kirillov \& Kirillova, 2013), Ukraine (PL 0.7\%, II 5-54) (Iskova et al., 1995; Yuskiv \& Melnychuk, 2020), Turkey - Marmara (Merdivenci, 1967; Gicik \& Arslan, 2003), Uzbekistan (Shakarbayev et al., 2012), Azerbaijan - Masally, Shabran regions (Shirinov, 1961; Vahidova, 1978), own data - Nakhchivan AR - Babek region (PL 2.2\%, II 2-3).

Distribution: Europe, Asia, Africa, North America.

24. Hypoderaeum dingeri Lie, 1964

First intermediate hosts: Mollusca-Gastropoda. 
Second intermediate hosts: Mollusca - Gastropoda; Amphibia Rhacophoridae.

Final hosts: Aves - Anseriformes, Columbiformes.

Site in host: small intestine.

Locality: Malaysia (McDonald, 1969).

Distribution: Asia.

Genus: Neoacanthoparyphium Yamaguti, 1958

25. Neoacanthoparyphium echinatoides Filippi, 1854

First intermediate hosts: Mollusca-Gastropoda.

Second intermediate hosts: Mollusca - Gastropoda, Bivalvia.

Final hosts: Aves - Anseriformes, Columbiformes, Galliformes, Cha-

radriiformes, Passeriformes; Mammalia - Rodentia, Lagomorpha.

Site in host: small intestine.

Locality: Slovakia (McDonald, 1969).

Distribution: Europe.

26. Neoacanthoparyphium petrovi Nevostrueva, 1953

First and second intermediate hosts: Mollusca-Gastropoda.

Final hosts: Aves - Anseriformes.

Site in host: small intestine.

Locality: Russia-Moscow region (Ryzhikov, 1967).

Distribution: Europe, Asia.

Genus: Petasiger Dietz, 1909 (Syn.: Paryphostomum Dietz, 1909)

27. Paryphostomum novum Verma, 1936

First intermediate hosts: unknown.

Second intermediate hosts: Mollusca - Gastropoda.

Final hosts: Aves - Anseriformes.

Site in host: intestine.

Locality: Far East (McDonald, 1969), Azerbaijan - Shabran region (PL 0.6\%, II 1-2) (Shirinov, 1961), own data - Lenkaran region (PL $0.86 \%$, II 1$)$.

Distribution: Europe, Asia.

28. Paryphostomum pentalobum Verma, 1936

First intermediate hosts: unknown.

Second intermediate hosts: unknown.

Final hosts: Aves - Anseriformes, Charadriiformes.

Site in host: intestine.

Locality: India (McDonald, 1969), Azerbaijan - Shabran region (EI 0.45\%, II 1) (Shirinov, 1961; Vahidova, 1978).

Distribution: Asia.

29. Petasiger radiatus (Dujardin, 1845) Tkach, Kudlai, Kostadinova, 2015

First intermediate hosts: Mollusca - Gastropoda.

Second intermediate hosts: Pisces.

Final hosts: Aves - Anseriformes, Podicipediformes, Pelecaniformes, Gruiformes.

Site in host: intestine.

Locality: Pakistan (McDonald, 1969)

Distribution: Europe, Asia, Africa, Australia.

Family: Philophthalmidae Looss, 1899

Genus: Philophthalmus Looss, 1899

30. Philophthalmus cupensis Richter, Vrazic \& Aleraj, 1953

Intermediate hosts: Mollusca-Gastropoda.

Final hosts: Aves - Anseriformes.

Site in host: eye - under nictitating membrane.

Locality: Serbia (Richter et al., 1953; McDonald, 1969).

Distribution: Europe.

31. Philophthalmus gralli Mathis \& Leger, 1910

Intermediate hosts: Mollusca-Gastropoda.

Final hosts: Aves - Anseriformes, Galliformes, Gruiformes, Struthioniformes; Mammalia - Lagomorpha, Rodentia and human (Dissanaike \& Bilimoria, 1958; Mimori et al., 1982).

Site in host: eye - conjunctival sac.

Locality: China (McDonald, 1969) and Taiwan (Kingston, 1984).
Distribution: Europe, Asia, Oceania, North America, Africa.

32. Philophthalmus hovorkai Busa, 1956

Intermediate hosts: Mollusca - Gastropoda.

Final hosts: Aves - Anseriformes, Galliformes, Falconiformes, Columbiformes, Ciconiiformes, Gruiformes, Charadriiformes; Mammalia Rodentia, Lagomorpha and humans (Kanev et al., 1993).

Site in host: eye - conjunctival sac.

Locality: Slovakia - Jahodna, Senne, Sturovo (Sitko et al., 2006), Uzbekistan - Tashkent (Shakarbayev et al., 2016), Austria - near Vienna (Kanev et al., 1993), Czech Republic and Bulgaria (Busa, 1956; Vassilev, 1962, 1973; Vassilev \& Denev, 1965, 1971), Germany (Siebold, 1835), France (Beneden, 1858)

Distribution: Europe, Asia.

33. Philophthalmus posaviniensis Richter, Wrazic, \& Aleraj, 1953 Intermediate hosts: Mollusca-Gastropoda.

Final hosts: Aves - Anseriformes.

Site in host: eye - under nictitating membrane.

Locality: Serbia (Richter et al.,1953; McDonald, 1969).

Distribution: Europe.

Family: Psilostomidae Looss, 1900

Genus: Psilochasmus Lühe, 1909

34. Psilochasmus longicirratus Skrjabin, 1913

First and second intermediate hosts: Mollusca-Gastropoda.

Final hosts: Aves - Anseriformes, Ciconiiformes, Columbiformes.

Site in host: small intestine - anterior region, caecum.

Locality: Kazakhstan (Petrochenko \& Kotelnikov, 1976).

Distribution: Europe, Asia, North America.

35. Psilochasmus oxyurus (Creplin, 1825) Lühe, 1909

First and second intermediate hosts: Mollusca-Gastropoda.

Final hosts: Aves - Anseriformes, Galliformes, Charadriiformes.

Site in host: small intestine - anterior region.

Locality: Brazil - State of Rio de Janeiro, Mage; State of Sao Paulo,

Sao Jose do Rio Preto (PL 20\%) (Berenice et al., 2007).

Distribution: Europe, Asia, Africa, North and South America.

Genus: Psilotrema Odhner, 1913

36. Psilotrema oligoon Linstow, 1887

Intermediate hosts: Mollusca - Gastropoda.

Final hosts: Aves - Anseriformes, Galliformes, Gruiformes, Colum-

biformes; Mammalia - Rodentia.

Site in host: small intestine.

Locality: Russia (McDonald, 1969).

Distribution: Europe, Asia.

37. Psilotrema simillimum Muhling, 1898

Intermediate hosts: Mollusca - Gastropoda

Final hosts: Aves - Anseriformes, Gruiformes; Mammalia - Rodentia.

Site in host: small intestine.

Locality: Germany (McDonald, 1969), Russia and Kazakhstan (Ryzhikov, 1967).

Distribution: Europe, Asia.

38. Psilotrema spiculigerum Muhling, 1898

Intermediate hosts: Mollusca-Gastropoda.

Final hosts: Aves - Anseriformes, Galliformes, Gruiformes; Mammalia-Rodentia

Site in host: small intestine.

Locality: Kazakhstan and Russia (Ryzhikov, 1967; Petrochenko \&

Kotelnikov, 1976).

Distribution: Europe, Asia.

Genus: Ribeiroia Travassos, 1939

39. Ribeiroia ondatrae (Price, 1931) Price, 1942

First intermediate hosts: Mollusca-Gastropoda.

Second intermediate hosts: Pisces-Centrarchidae, Poeciliidae, Percidae. 
Final hosts: Aves - Anseriformes, Falconiformes, Galliformes, Columbiformes, Passeriformes; Mammalia-Rodentia.

Site in host: proventriculus - in mucosa.

Locality: USA and Canada (Lapage, 1961; McDonald, 1969; Crompton \& Nesheim, 1976).

Distribution: North and South America.

Genus: Sphaeridiotrema Odhner, 1913

40. Sphaeridiotrema globulus (Rudolphi, 1819) Odhner, 1913

Intermediate hosts: Mollusca-Gastropoda.

Final hosts: Aves - Anseriformes, Gruiformes, Charadriiformes.

Site in host: small intestine.

Locality: UK (Crompton \& Nesheim, 1976).

Distribution: Europe, Asia, North America.

Family: Typhlocoelidae Harrah, 1922

Genus: Tracheophilus Skrjabin, 1913

41. Tracheophilus sisowi Skrjabin, 1913

Intermediate hosts: Mollusca-Gastropoda.

Final hosts: Aves - Anseriformes, Charadriiformes.

Site in host: trachea, bronchi, oronasal passage, air sacs.

Locality: Russia - along the Volga River (Ryzhikov, 1967; Petrochenko \& Kotelnikov, 1976; Kirillov \& Kirillova, 2013).

Distribution: Europe, Asia, Africa, North and South America, Australia.

Family: Heterophyidae Leiper, 1909

Genus: Cercarioides Witenberg, 1929

42. Cercarioides aharoni Witenberg, 1929

First and second intermediate hosts: unknown.

Final hosts: Aves - Anseriformes, Charadriiformes.

Site in host: intestine.

Locality: Egypt and Ukraine (Gohar, 1935; McDonald, 1969; Smogorzhevskaya, 1976)

Distribution: Europe, Asia, Africa.

Genus: Cryptocotyle Lühe, 1899

43. Cryptocotyle concava Creplin, 1825

First intermediate hosts: Mollusca-Gastropoda.

Second intermediate hosts: Pisces - Atherinidae, Gobiidae, Catostomidae, Gasterosteidae, Gobiidae, Mugilidae, Mullidae.

Final hosts: Aves - Anseriformes, Gaviiformes, Podicipediformes, Pelecaniformes, Ciconiiformes, Galliformes, Charadriiformes, Columbiformes, Strigiformes; Mammalia - Lagomorpha, Rodentia, Carnivora, Artiodactyla.

Site in host: intestine, caecum.

Locality: Russia (Ryzhikov, 1967).

Distribution: Europe, Asia, North America.

Genus: Pygidiopsis Looss, 1907

44. Pygidiopsis genata Looss, 1907

First intermediate hosts: Mollusca-Gastropoda.

Second intermediate hosts: Pisces - Atherinidae, Mugilidae, Cichilidae, Cyprinidae, Poeciliidae.

Final hosts: Aves - Anseriformes, Podicipediformes, Pelecaniformes, Ciconiiformes, Falconiformes, Galliformes, Charadriiformes, Columbiformes, Strigiformes; Mammalia - Lagomorpha, Rodentia, Carnivora and humans (Boulos et al.,1981).

Site in host: intestine.

Locality: Ukraine (Smogorzhevskaya, 1976).

Distribution: Europe, Asia, Africa, North America.

Family: Opisthorchiidae Looss, 1899

Genus: Metorchis Looss, 1899

45. Metorchis orientalis Tanabe, 1920

First intermediate hosts: Mollusca-Gastropoda.

Second intermediate hosts: Pisces - Cyprinidae.
Final hosts: Aves - Anseriformes, Podicipediformes, Ciconiiformes, Galliformes, Strigiformes; Mammalia - Rodentia, Carnivora and humans (Li et al., 2010).

Site in host: Gall bladder, bile ducts of liver.

Locality: China - Tsingkiangpu, Kiangsu (Zhan et al., 2017).

Distribution: Asia, North America.

46. Metorchis taiwanensis Morishita \& Tsuchimochi, 1925

First intermediate hosts: unknown.

Second intermediate hosts: Pisces - Cyprinidae.

Final hosts: Aves - Anseriformes, Falconiformes, Galliformes, Gruiformes.

Site in host: gall bladder, bile ducts of liver.

Locality: Russia (Ryzhikov, 1967).

Distribution: Asia.

47. Metorchis xanthosomus (Creplin, 1846) Braun, 1902

First intermediate hosts: Mollusca-Gastropoda.

Second intermediate hosts: Pisces - Cobitidae, Gasterosteidae, Nemachilidae, Cyprinidae.

Final hosts: Aves - Anseriformes, Gaviiformes, Podicipediformes, Pelecaniformes, Falconiformes, Galliformes, Gruiformes, Passeriformes; Mammalia-Carnivora.

Site in host: gall bladder, bile ducts of liver, intestine.

Locality: China (McDonald, 1969).

Distribution: Asia, Europe.

\section{Genus: Opisthorchis Blanchard, 1895}

48. Opisthorchis simulans Looss, 1896

First and second intermediate hosts: unknown.

Final hosts: Aves - Anseriformes, Ciconiiformes, Falconiformes, Gruiformes.

Site in host: bile ducts of liver.

Locality: Ukraine (Smogorzhevskaya, 1976; Petrochenko \& Kotelnikov, 1976; McDonald, 1969).

Distribution: Europe, Asia, Africa, North America.

Family: Notocotylidae Luhe, 1909

Genus: Catatropis Odhner, 1905

49. Catatropis verrucosa Froelich, 1789

First intermediate hosts: Mollusca-Gastropoda.

Second intermediate hosts: Oligocheata - Clitellata.

Final hosts: Aves - Anseriformes, Galliformes, Gruiformes, Charadriiformes.

Site in host: intestine, caecum.

Locality: Russia - Bashkortostan, Dagestan, Ural and Amur regions, along the Volga River, North Caucasus (Marzhokhova \& Zhigunova, 2008; Kirillov \& Kirillova, 2013), Turkey - Marmara (Merdivenci, 1967; Gicik \& Arslan, 2003), Czech Republic (Barus et al., 1977), Uzbekistan (Shakarbayev et al., 2012), Azerbaijan - Shabran, Khachmaz regions (Shirinov, 1961).

Distribution: Europe, Asia, Africa, North America.

Genus: Notocotylus Diesing, 1839

50. Notocotylus attenuatus (Rudolphi, 1809) Kossack, 1911

Intermediate hosts: Mollusca-Gastropoda.

Final hosts: Aves - Anseriformes, Galliformes, Gruiformes, Charadriiformes; Mammalia - Rodentia.

Site in host: caecum, large intestine.

Locality: Czech Republic and Slovakia (PL 0.3\%) (Busta, 1980; Sitko et al., 2006), Russia - along the Volga River, Dagestan, North Caucasus (Marzhokhova \& Zhigunova, 2008; Kirillov \& Kirillova, 2013), England (Soliman, 1955; Griffiths et al., 1976), Uzbekistan (Shakarbayev et al., 2012), Ukraine (PL 0.07\%, II 4-10) (Smogorzhevskaya, 1976; Iskova et al., 1995), Turkey - Marmara (Merdivenci, 1967), Iraq - Ninevah governorate (Al-Taee et al., 2011), Azerbaijan - Astara, Shabran, Khachmaz regions (PL 2.3\%, II 2-10) (Shirinov, 1961; Vahidova, 1978) own data - Khudat, Bilasuvar regions, Nakhchivan AR - Julfa region (PL $1.1 \%$, II 7-12). 
Distribution: Europe, Asia, North and South America, Australia.

51. Notocotylus ephemera Nitzsch, 1817

Intermediate hosts: Mollusca-Gastropoda.

Final hosts: Aves-Anseriformes, Galliformes.

Site in host: caecum.

Locality: Germany-Berlin (McDonald, 1969).

Distribution: Europe.

52. Notocotylus imbricatus Looss, 1893

Intermediate hosts: Mollusca-Gastropoda.

Final hosts: Aves - Anseriformes, Galliformes.

Site in host: caecum.

Locality: China (Chang-Tung, 1937; Gower, 1939; McDonald, 1969).

Distribution: Europe, Asia, North America.

53. Notocotylus parviovatus Yamaguti, 1934

Intermediate hosts: Mollusca-Gastropoda.

Final hosts: Aves - Anseriformes.

Site in host: caecum, intestine.

Locality: Ukraine (Iskova et al., 1995; Yuskiv \& Melnychuk, 2020),

Russia - along the Volga River (Kirillov \& Kirillova, 2013), Uzbekistan

(PL 3.1\%, II 1-8) (Shakarbayev et al., 2012), Azerbaijan - Garabakh

(Vahidova, 1978).

Distribution: Europe, Asia.

54. Notocotylus seineti Fuhrmann, 1919

First intermediate hosts: Mollusca-Gastropoda.

Second intermediate hosts: Mollusca - Gastropoda; Artrophoda -

Cyprididae.

Final hosts: Aves - Anseriformes.

Site in host: caecum, intestine.

Locality: Germany (Heinemann, 1936).

Distribution: Europe, North America.

55. Notocotylus stagnicolae Herber, 1942

Intermediate hosts: Mollusca-Gastropoda.

Final hosts: Aves - Anseriformes, Galliformes, Charadriiformes;

Mammalia-Rodentia.

Site in host: caecum.

Locality: USA (McDonald, 1969).

Distribution: North America.

56. Notocotylus thienemanni Szidat \& Szidat, 1933

Intermediate hosts: Mollusca-Gastropoda.

Final hosts: Aves - Anseriformes.

Site in host: caecum.

Locality: Germany (Heinemann, 1936).

Distribution: Europe.

Genus: Paramonostomum Lühe, 1909

57. Paramonostomum alveatum (Mehlis, 1846) Lühe, 1909

First intermediate hosts: Mollusca-Gastropoda.

Second intermediate hosts: Mollusca - Gastropoda, Bivalvia; Crusta-

cea-Gammaridae.

Final hosts: Aves - Anseriformes, Galliformes.

Site in host: caecum, intestine, cloaca.

Locality: Ukraine (Smogorzhevskaya, 1976).

Distribution: Europe, Asia, North America.

58. Paramonostomum bucephalae Yamaguti, 1935

First intermediate hosts: Mollusca-Gastropoda.

Second intermediate hosts: Mollusca - Gastropoda, Bivalvia; Crustacea-Gammaridae.

Final hosts: Aves - Anseriformes.

Site in host: caecum.

Locality: Ukraine (McDonald, 1969; Smogorzhevskaya, 1976).

Distribution: Europe, Asia, North America.

Genus: Uniserialis Beverley-Burton, 1958

59. Uniserialis gippyensis Beverley-Burton, 1958

Intermediate hosts: Mollusca-Gastropoda.
Final hosts: Aves - Anseriformes.

Site in host: caecum, bursa of Fabricius.

Locality: New Zealand - Ashburton, Avon R., Banks Peninsula,

L. Coleridge, Otago (Rind, 1974; Weekes, 1982; McKenna, 2010).

Distribution: Europe, Oceania.

60. Uniserialis tadornae Bisset 1977

Intermediate hosts: Mollusca-Gastropoda.

Final hosts: Aves-Anseriformes.

Site in host: caecum.

Locality: New Zealand (Bisset, 1977; Weekes, 1982).

Distribution: Oceania.

Family: Zygocotylidae Ward, 1917

Genus: Zygocotyle Stunkard, 1917

61. Zygocotyle lunata Diesing, 1836

Intermediate hosts: Mollusca - Gastropoda.

Final hosts: Aves - Anseriformes, Galliformes, Charadriiformes;

Mammalia - Rodentia, Artiodactyla.

Site in host: caecum, intestine.

Locality: France (Travassos, 1933).

Distribution: Asia, Europe, Africa, North and South America.

Family: Prosthogonimidae Lühe, 1909

Genus: Prosthogonimus Lühe, 1899 (Syn.: Schistogonimus Lühe, 1909)

62. Prosthogonimus anatinus Markov, 1903

First intermediate hosts: Mollusca-Gastropoda.

Second intermediate hosts: Insecta - Aeshnidae.

Final hosts: Aves - Anseriformes, Galliformes, Gruiformes, Charadriiformes.

Site in host: bursa of Fabricius.

Locality: USA - Michegan (Gower,1939).

Distribution: Europe, Asia, North America.

63. Prosthogonimus cuneatus (Rudolphi, 1809) Braun, 1901

First intermediate hosts: Mollusca - Gastropoda.

Second intermediate hosts: Insecta - Aeshnidae, Cordiliidae, Libelliludae.

Final hosts: Aves - Anseriformes, Podicipediformes, Pelecaniformes, Ciconiiformes, Falconiformes, Galliformes, Gruiformes, Charadriiformes, Piciformes, Passeriformes.

Site in host: bursa of Fabricius, cloaca, oviduct, rectum.

Locality: Ukraine - Odesa region (Smogorzhevskaya, 1976; Iskova et al., 1995), Russia - Dagestan, Bashkortostan, along the Volga River, North Caucasus, Rostov, Moscow, Omsk regions (Petrochenko \& Kotelnikov, 1976; Marzhokhova \& Zhigunova, 2008; Kirillov \& Kirillova, 2013).

Distribution: Europe, Asia, Africa, North and South America.

64. Prosthogonimus ovatus (Rudolphi, 1803) Lühe, 1899

First intermediate hosts: Mollusca-Gastropoda.

Second intermediate hosts: Insecta - Aeshnidae, Cordiliidae, Libelliludae.

Final hosts: Aves - Anseriformes, Podicipediformes, Pelecaniformes, Ciconiiformes, Falconiformes, Galliformes, Gruiformes, Charadriiformes, Cuculiformes, Strigiformes, Coraciiformes, Piciformes, Passeriformes.

Site in host: bursa of Fabricius, oviduct, cloaca, large intestine.

Locality: Uzbekistan (Shakarbayev et al., 2012), Ukraine (Smogorzhevskaya, 1976; Iskova et al., 1995), Russia - Krasnoyarsk region and Kazakhstan (Petrochenko \& Kotelnikov, 1976).

Distribution: cosmopolitan.

65. Schistogonimus rarus Braun, 1901

First intermediate hosts: Mollusca-Gastropoda.

Second intermediate hosts: Insecta-Coenagrionidae, Lestidae.

Final hosts: Aves - Anseriformes, Galliformes, Gruiformes, Charadriiformes.

Site in host: bursa of Fabricius.

Locality: Russia - Dagestan (Ryzhikov, 1967).

Distribution: Europe, Asia, North America. 


\section{Discussion}

Of the 65 species of trematodes parasitizing in domestic geese, 44 species were recorded in Europe, 26 species in Asia, 6 species in North America, 1 species in South America, 1 species in Africa and 2 species in Oceania. Three species registered in domestic geese (E. recurvatum, E. revolutum, $P$. ovatus) are cosmopolitan parasites. In the territory of Europe, domestic geese are mainly parasitized by species belonging to the families Strigeidae, Cyathocotylidae, Cyclocoelidae, Echinochasmidae, Echinostomatidae, Philophthalmidae, Psilostomidae, Typhlocoelidae, Heterophyidae, Notocotylidae, Zygocotylidae, Prosthogonimidae, in Asia - Echinostomatidae, Psilostomidae, Opisthorchiidae, Philophthalmidae, in North and South America - Strigeidae, Schistosomatidae, Psilostomidae, Notocotylidae, Prosthogonimidae, in Africa - Heterophyidae, in Oceania - Notocotylidae. The greatest species diversity is characterized by the families Echinostomatidae (17 species) and Notocotylidae (12 species), and the smallest Echinochasmidae, Typhlocoelidae, Zygocotylidae (1 species for each). Eleven species of digenetic flukes were recorded in domestic geese (C. mutabile, E. grande, E. paraulum, E. revolutum, E. robustum, $H$. conoideum, $P$. novum, $P$. pentalobum, $C$. verrucosa, $N$. attenuatus, N. parviovatus) on the territory of Azerbaijan. Four of them (E. revolutum, $H$. conoideum, P. novum, $N$. attenuatus) were also noted in the course of our helminthological studies. The distribution of all 11 species of trematodes on the territory of the republic is shown in Figure 1. The trematode C. mutabile was recorded in domestic ducks only in Azerbaijan.

Most species of trematodes were found in the north-eastern part (total 9 species) and southern part (total 5 species) of Azerbaijan. In the abovementioned regions, there are favourable conditions for the spread of mollusks, intermediate hosts of digenetic flukes involved in the development of trematodes. The aforementioned areas are located on the shores of the Caspian Sea, and most of the country's rivers flow through these areas and flow into the sea.

Eighteen species of digenetic flukes (E. recurvatum, E. lindoense, E. miyagawai, E. revolutum, $N$. echinatoides, $P$. hovorkai, $P$. gralli, $P$. oligoon, $P$. simillimum, $P$. spiculigerum, $R$. ondatrae, $C$. concava, P. genata, M. orientalis, $M$. xanthosomus, $N$. attenuatus, $N$. stagnicolae, $Z$. lunata) parasitize both birds and mammals (Rodentia, Carnivora, Lagomorpha, Artiodactyla). And eleven species (6 species from the family Echinostomatidae - E. recurvatum, E. lindoense, E. paraulum, E. revolutum, E. miyagawai, H. conoideum, 2 species from the family Philophthalmidae - P. gralli, P. hovorkai, 1 species from the family Strigeidae C. cornutus, 1 species from the family Heterophyidae $-P$. genata, 1 species from the family Opisthorchiidae $-M$. orientalis) of trematodes are of medical importance, registered in humans.

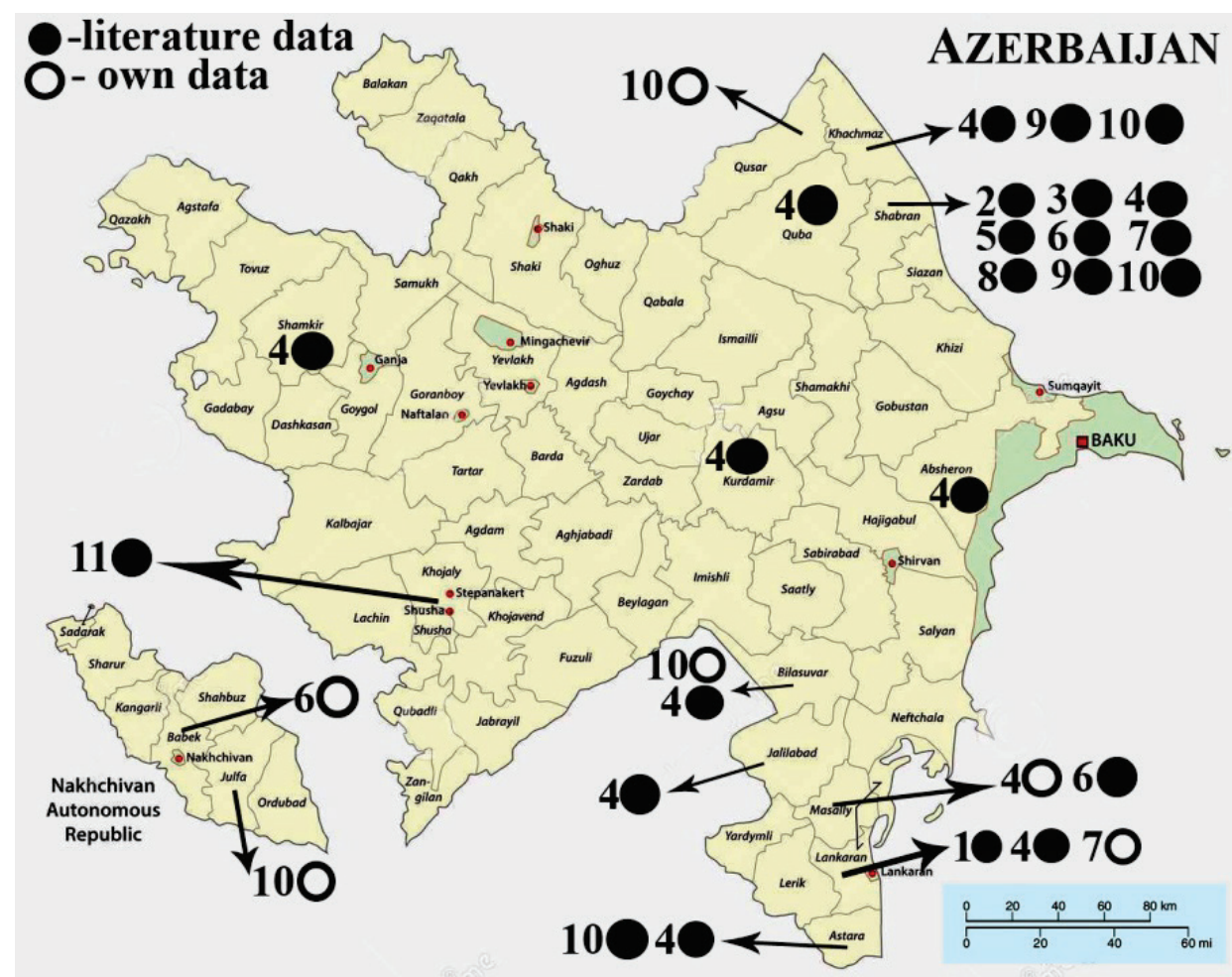

Fig. 1. Map of the areas of occurrence of trematodes in domestic geese in Azerbaijan: 1-Cyclocoelum mutabile, 2-Echinostoma grande, 3-Echinostoma paraulum, 4-Echinostoma revolutum, 5-Echinostoma robustum, 6-Hypoderaeum conoideum, 7-Paryphostomum novum, 8-Paryphostomum pentalobum, 9-Catatropis verrucosa, 10-Notocotylus attenuatus, 11-Notocotylus parviovatus

\section{Conclusion}

Own research and analysis of extensive literature data showed that the taxonomic composition of the Trematoda of domestic geese consists of 2 classes, 14 families, 30 genera and 65 species (Europe - 44, Asia - 26, North and South America - 7, Africa-1, Oceania-2, Cosmopolitan -3). The greatest species diversity is typical for the families Echinostomatidae (17 species) and Notocotylidae (12 species).

On the territory of Azerbaijan, among domestic geese, 11 species of digenetic flukes have been registered. Four of them (E. revolutum, $H$. conoideum, $P$. novum, $N$. attenuatus) were also noted during our helminthological studies. The trematode $C$. mutabile has been registered in domestic ducks only in Azerbaijan. Most of the trematode species were found in the northeastern ( 9 species in total) and in the southern part ( 5 species in total) of Azerbaijan.

\section{References}

Aghayeva, Z. T. (2018). Azerbaycanin muxtelif rayonlarinda gaz (Anser anser dom.) ve ordeklerin (Anas platherhynchos dom.) helmintlerin bio-ekoloji xususiyyetlerinin oyrenilmesi [Study of bio-ecological features of helminths of goose (Anser anser dom.) and duck (Anas platherhynchos dom.) in different regions of Azerbaijan]. Elm, Baku (in Azerbaijanian).

Al-Taee, A. F., Mohammed, R. G., \& Mohammed, N. H. (2011). Diagnosis of some helminthic eggs in faces of ducks and geese in Nineveh governorate, Iraq. Iraqi J Vet Sci., 25(1), 5-10.

Barus, V., Micolasek, A., \& Busta, J. (1977). Influence of breeding technology on helminthfauna of geese (Anser anser f. dom.). Folia Parasitologica, 24, 305-314.

Beneden, P. J. V. (1858). Mémoire sur les vers intestinaux [Dissertation about intestinal worms]. PI Brailliere \& Fils, Paris (in France).

Bezubik, B. (1956). Helmintofauna dzikich kaczek (podrodzina Anatinae) Woj Lubelskiego i Białostockiego [Helminthological fauna in wild ducks (subfamily 
Anatinae) in the Lublin and Bialystok Regions]. Wiadomosci Parazytologiczne, 2, 267-268 (in Polish).

Bhaibulaya, M., Charoenlarp, P., \& Harinasuta, C. (1964). Report of cases Echinostoma malayanum and Hypoderaeum conoideum in Thailand. Journal of the Medical Association of Thailand, 47, 720-730.

Bisset, S. A. (1977). Notocotylus tadornae n. sp. and Notocotylus gippyensis (Beverley-Burton, 1958) (Trematoda: Notocotylidae) from waterfowl in New Zealand: Morphology, life history and systematic relations. J Helminthol., 51, 365 372.

Boulos, L. M., Abdou, L. A., \& Girgis, R. S. (1981). Histopathological and histochemical studies on experimentally infected hamsters with Pygidiopsis genata. Journal of the Egyptian Society of Parasitology, 11, 67-76.

Brant, S. V., \& Loker, E. S. (2009). Molecular systematics of the avian schistosome genus Trichobilharzia (Trematoda: Schistosomatidae) in North America. Journal of Parasitology, 95(4), 941-963.

Bray, R. A., Gibson, D. I., \& Jones, A. (2008). Keys to the Trematoda. Vol. 3. CAB International and Natural History Museum, London.

Busa, V. (1956). Novy trematod Philophthalmus (Tubolecithalmus) hovorkai n. sp. u husi domacej (Anser anser dom.) [New trematode Philophthalmus (Tubolecithalmus) hovorkai n. sp. in domestic geese (Anser anser dom.)]. Biologia, 2, 151-258 (in Chech).

Busta, J. (1980). Helminti husích brojlerü vykrmovaných ve výbězích [Helminths in broiler geese fattened in runs]. Veterinary Medicine (Prague), 25(12), 717-723 (in Czech).

Carney, W. P. (1991). Echinostomiasis - a snail-borne intestinal trematode zoonosis. The Southeast Asian Journal of Tropical Medicine and Public Health, 22, 206-211.

Carney, W. P., Sudono, M., \& Purnomo, A. (1980). Echinostomiasis: A disease that disappeared. Tropical and Geographical Medicine, 32, 106-111.

Chang-Tung, K. (1937). On a new trematode parasite from the Peking area. Peking Natural History Bulletin, 12(1), 39-41.

Crompton, D. W. T., \& Nesheim, M. C. (1976). Host-parasite relationships in the alimentary tract of domestic birds. Advances in Parasitology, 14, 95-194.

Dissanaike, A. S., \& Bilimoria, D. P. (1958). On an infection of a human eye with Philophthalmus sp. in Ceylon. Journal of Helmintology, 32, 115-118.

Dubinina, M. N. (1971). Parazitologichekoye issledovaniye ptits [Parasitological study of birds]. Nauka, Leningrad (in Russian).

Fernandes, B. M. M., \& Kohn, A. (2014). South American trematodes parasites of birds and mammals. Oficina de Livros, Rio de Janeiro.

Fernandes, M. M. B., Menezes, C. R., Tortelly, R., Noronha, D., \& Pinto, M. R. (2007). First report of the digenetic trematode Psilochasmus oxyurus (Creplin) in the domestic goose, Anser anser (Linnaeus) in South America. Rev. Bras. Zool., 24(2), 520-522.

Gibson, I. D., Jones, A., \& Bray, R. A. (2002). Keys to the trematoda. Vol. 1. CABI/ The Natural History Museum, London.

Gicik, Y., \& Arslan, M. O. (2003). The prevalence of helminths in the alimentary tract of geese (Anser anser domesticus) in Kars District, Turkey. Vet Res Commun., 27, 391-395.

Gohar, N. (1935). Liste de Trematodes parasites et de leurs hotes vertebres signales dans la vallee du Nil. Ann. Parasit., 13(1), 80-90.

Gower, W. C. (1939). Host-parasite catalogue of the helminths of ducks. American Midland Naturalist, 22(3), 580-628.

Griffiths, H. J., Gonder, E., \& Pomeroy, B. S. (1976). An outbreak of trematodiasis in domestic geese. Avian Diseases, 20(3), 604-606.

Heinemann, E. (1936). Die Parasiten des Hausgeflugels. 6. Parasitische Wiirmer als Ursache eines Gansesterbens. Archiv fur Geflugelkunde, 10, 322-336.

Iskova, N. I., Sharpilo, V. P., Sharpilo, L. D., \& Tkach, V. V. (1995). Katalog gel'mintov pozvonochnykh Ukrainy. Trematody nazemnykh pozvonochnykh [Catalogue of helminths of vertebraes in Ukraine. Trematodes of terrestrial vertebrates]. Institute of Zoology NAS of Ukraine, Kiev (in Russian).

Jones, A., Bray, R. A., \& Gibson, D. I. (2005). Keys to the Trematoda. Volume 2. CABI and Natural History Museum, London.

Kanev, İ., Nollen, P., Vassilev, I., Radev, V., \& Dimitrov, V. (1993). Redescription of Philophthalmus lucipedus (Rudolphi, 1819) (Trematoda: Philophthalmidae) with a discussion of its identity and characteristics. Annalen des Naturhistorischen Museums in Wien, 94/95B, 11-34.

Kingston, N. (1984). Trematodes. Diseases of poultry. Iowa University Press, Ames.

Kirillov, A. A., \& Kirillova, N. Y. (2013). Trematodes of birds (Aves) of the Middle Volga Region. 2. Orders Plagiorchiida, Renicolida, Strigeida and Schistosomatida. Parasitologiia, 47(2), 136-177.

Lapage, G. (1961). A list of the parasitic protozoa, helminths and arthropoda recorded from species of the family Anatidae (ducks, geese and swans). Parasitology, $51,1-109$.

Li, T., He, S., Zhao, H., Zhao, G., \& Zhu, X. Q. (2010). Major trends in human parasitic diseases in China. Trends in Parasitology, 26(5), 264-270.

Liu, D., Zhuo, Z., Tao, J., \& Xu, J. (2018). A case report of Apatemon gracilis (Szidat, 1928) infection in domestic geese in mainland China. Turk J Vet Anim Sci, 42, 139-142.
Lu, S. C. (1982). Echinostomiasis in Taiwan. International Journal of Zoonoses., 9, 33-38.

Macy, R. W., Moore, D. J., \& Price, W. S. (1955). Studies on dermatitis-producing schistosomes in the Pacific Northwest, with special reference to Trichobilharzia oregonensis. Trans. Amer. Mier. Soc., 74(3), 235-251.

Mao, S. P. (1991). Protozoan and helminth parasites of humans in mainland China. International Journal for Parasitology, 21(3), 347-351.

Marzhokhova, L. M., \& Zhigunova, A. A. (2008). Parasite fauna of domestic ducks of the North Caucasus and its ecological and epizootic characteristics. Russian Parasitological Journal, 1, 1-12.

McDonald, M. E. (1969). Catalogue of helminths of waterfowl (Anatidae). United States National Government Publication, Washington.

McKenna, P. (2010). An updated checklist of helminth and protozoan parasites of birds in New Zealand. Webmed Central Parasitology, 1(9).

Merdivenci, A. (1967). Türkiye'nin Marmara bölgesinde evcil tavuk, hindi, ördek ve kazlarda görülen trematod, sestod ve nematodlara dair araştirmalar [Studies on trematodes, cestodes and nematodes in domestic chickens, turkeys, ducks and geese in the Marmara region of Turkey]. Kutulmush, İstanbul (in Turkish).

Mimori, T., Hirai, H., Kifune, T., \& Inada, K. (1982). Philophthalmus sp. (Trematoda) in a human eye. American Journal of Tropical Medicine and Hygiene, 31, 859-861.

Olson, P. D., Cribb, T. H., Tkach, V. V., Bray, R. A., \& Littlewood, D. T. J. (2003). Phylogeny and classification of the Digenea (Platyhelminthes: Trematoda). International Joumal for Parasitology, 33, 733-755.

Petrochenko, V. I., \& Kotelnikov, G. A. (1976). Gel'mintozy ptits [Helminthiasis of birds]. Kolos, Moscow (in Russian).

Richter, S., Vrazic, O., \& Aleraj, Z. (1953). Filoftalmoza domace guske. Veterinarski Arhiv, 23, 193-205.

Rind, S. (1974). Some helminth parasites of freshwater birds from the South Island, New Zealand, with particular reference to trematodes of ducks. Mauri Ora, 2, $139-146$.

Ryzhikov, K. M. (1967). Opredelitel' gel'mintov domashnikh vodoplavayushchikh ptits [Key to helminths of domestic waterfowl]. Nauka, Moscow (in Russian).

Rzayev, F. H. (2008). Ev su quşlarının helmintozları [Helminthiasis of domestic waterfowl]. Science and Life, 2, $44-45$ (in Azerbaijanian).

Rzayev, F. H. (2021). Cestodes (Plathelminthes: Cestoda) of domestic waterfowl. Advances in Biology and Earth Sciences, 6(2), 133-141.

Rzayev, F. H., \& Ibrahimova, N. E. (2015). Sravnitel'naya kharakteristika gel'mintofauny domashnikh vodoplavayushchikh ptits yugo-vostochnoy chasti Azerbaydzhana [Comparative characteristics of helminthofauna of domestic water birds of the southeast of Azerbaijan]. Ecological Bulletin, 32, 101-106 (in Russian).

Rzayev, F. H., Nasirov, A. M., \& Gasimov, E. K. (2021). A systematic review of tapeworms (Plathelminthes, Cestoda) of domestic ducks (Anas platyrhynchos dom.). Regulatory Mechanisms in Biosystems, 12(2), 353-361.

Rzayev, F. H., Seyidbeyli, M. I., Maharramov, S. H., \& Gasimov, E. K. (2020). Formy i ultrastruktumye osobennosti lateral'nykh kryl'ev gel'minta Trichostrongylus tenuis Mehlis, 1846 (Nematoda: Trichostrongylidae) [Forms and ultrastructural features of the lateral alae of the helminth Trichostrongylus tenuis Mehlis, 1846 (Nematoda: Trichostrongylidae)]. The Journal of V. N. Karazin Kharkiv National University, 34, 112-119 (in Russian).

Seyidbeyli, M. İ., \& Rzayev, F. H. (2018). Helminth fauna of waterfowl poultry in the territory of Babek region of Nakhcivan AR. Journal of Entomology and Zoology Studies, 6(1), 1668-1671.

Seyidbeyli, M. I., Rzayev, F. H., \& Gasimov, E. K. (2020). Ul'trastrukturnye osobennosti kozhno-muskul'nogo meshka gel'minta Trichostrongylus temuis (Mehlis, 1846) (Nematoda: Trichostrongylidae) [Ultrastructural features of the body wall of the helminth Trichostrongylus tenuis (Mehlis, 1846) (Nematoda: Trichostrongylidae)]. Parasitologiia, 54(5), 402-412 (in Russian).

Shakarbayev, E. B., Akramova, F. D., \& Azimov, D. A. (2012). Trematody - parazity pozvonochnykh Uzbekistana (struktura, funktsionirovaniye i bioekologii) [Trematodes - parasites of vertebrates in Uzbekistan (structure, functioning and bioecology)]. Chinor ENK, Tashkent (in Russian).

Shakarbayev, E. B., Akramova, F. D., \& Azimov, D. A. (2016). Melanoides kainarensis - new intermediate host of trematodes Philophthalmus lucipedus (Trematoda, Philophthalmidae). Russian Journal of Parasitology, 36(2), 183-191.

Shirinov, N. M. (1961). Gel'mintofauna i gel'mintozy domashnikh vodoplavayushchikh ptits Azerbaydzhanskoy SSR i ispytaniye piperazin-sul'fata pri ganguleterakidoze [Helminth fauna and helminthiasis of domestic waterfowl of the Azerbaijan SSR and the test of piperazine sulfate in ganguleteracidosis]. Elm, Baku (in Russian).

Siebold, C. T. V. (1835). Helminthologische Beiträge [Helminthological contributeons]. Archiv für Naturgeschichte, Nicolai'sche Buchhandlung, 1(1), 45-83 (in German).

Sitko, J., Faltýnková, A., \& Scholz, T. (2006). Checklist of the Trematodes (Digenea) of birds of the Czech and Slovak Republics. Academia, Praha.

Skrjabin, K. I. (1928). Metod polevykh gel'mintologycheskikh vskrytiy pozvonochnykh, vklyuchaya cheloveka [The method of field helminthological examina- 
tion of vertebrates, including humans]. Moscow State University, Moscow (in Russian)

Smogorzhevskaya, L. A. (1976). Gel'minty vodoplavayushchikh i bolotnych ptits fauny Ukrainy [Helminths of waterfowl and wading birds of the fauna of Ukraine]. Nauka Dumka, Kiev (in Russian).

Sohn, W. M., Chai, J. Y., Yong, T. S., Eom, K. S., Yoon, C. Y., Sinuon, M., Socheat D., \& Lee, S. H. (2011). Echinostoma revolutum infection in children, Pursat Province, Cambodia. Emerg. Infect. Dis., 17(1), 117-119.

Soliman, K. N. (1955). Observations on some helminth parasites from ducks in Southern England. Journal of Helminthology, 29, 17-26.

Travassos, L. P. (1933). Observations sur Zygocotyle lunatum (Diesing, 1835) (Trematoda: Paramphistomidae). Comptes Rendus des Seances de la Societe de Biologie et des ses Filiales (Paris), 114(35), 958-959.

Vahidova, S. M. (1978). Gel'minty ptits Azerbaydzhana [Helminths of birds of Azerbaijan]. Elm, Baku (in Russian).

Vassilev, I. (1962). On the helminthofauna of domestic geese (Anser anser dom.) in Bulgaria. Izvestja Tsentra Khelmintologycheskoy Laboratorii (Sofia), 7, 11-17.

Vassilev, I. (1973). On the ecology of paratenogenetic stages of philophthalmids from Yugoslavia. Izvestja Tsentra Khelmintologycheskoy Laboratorii (Sofia), $16,25-29$.
Vassilev, I., \& Denev, I. (1965). Research into the life history of Philophthalmus sp. recovered from geese in Bulgaria. I. Zeitschrift Fur Parasitenkunde, 25, 320-329.

Vassilev, I., \& Denev, I. (1971). Research into the life history of Philophthalmus sp. recovered from geese in Bulgaria. II. Zeitschrift Fur Parasitenkunde, 37, 70-84.

Weekes, P. J. (1982). Checklist of helminth parasites of birds in New Zealand. New Zealand Journal of Zoology, 9, 451-460.

Yokogawa, M., Harinasuta, C., \& Charoenlarp, P. (1965). Hypoderaeum conoideum (Bloch, 1782) Dietz, 1909. A common intestinal fluke of man in Northeast Thailand. Japanese Journal of Parasitology, 14, 148-183.

Yu, S. H., \& Mott, K. E. (1994). Epidemiology and morbidity of food-borne intestinal trematode infections. Tropical Diseases Bulletin, 91, 125-152.

Yuskiv, I. D., \& Melnychuk, V. (2020). Spetsial'ni zaxody protigel'mintoznogo kompleksu za nayavnosti endoparazitoziv u gusej (Anser anser domesticus and Anser cygnoides domesticus Linnaeus, 1758) [Special measures of antihelmintoses complex in case of goose endoparasitoses (Anser anser domesticus \& Anser cygnoides domesticus Linnaeus, 1758)]. Bulletin of Poltava State Agrarian Academy, 1, 222-242 (in Ukrainian).

Zhan, X., Li, C., Wu, H., Sun, E., \& Zhu, Y. (2017). Investigation on the endemic characteristics of Metorchis orientalis in Huainan Area, China. Nutr Hosp., 34, 675-679. 